\title{
SGML Parser Validation Procedures
}

\author{
R. B. Wilson
}

U.S. DEPARTMENT OF COMMERCE Technology Administration National Institute of Standards and Technology

Gaithersburg, MD 20899

$Q C$

100

.456

N0.5538

1995 



\section{SGML Parser Validation Procedures}

\section{R. B. Wilson}

U.S. DEPARTMENT OF COMMERCE Technology Administration National Institute of Standards and Technology

Gaithersburg, MD 20899

January 1995

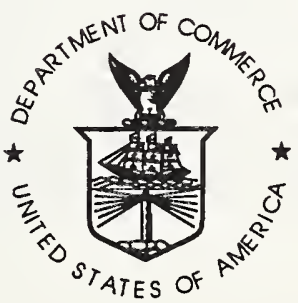

U.S. DEPARTMENT OF COMMERCE Ronald H. Brown, Secretary

TECHNOLOGY ADMINISTRATION Mary L. Good, Under Secretary for Technology NATIONAL INSTITUTE OF STANDARDS AND TECHNOLOGY

Arati Prabhakar, Director 



\title{
SGML PARSER VALIDATION PROCEDURES
}

\author{
by \\ Ronald B. Wilson \\ Systems and Software Technology Division \\ Computer Systems Laboratory \\ National Institute of Standards and Technology
}

January 1, 1995

FY93 CALS TASK 9.2.

"Develop a Conformance Testing

Service for ISO 8879."

Deliverables

Procedures for an ISO 8879

Conformance Testing Service

- ABSTRACT -

NIST has been tasked by the CALS Project Office to organize an SGML Conformance Testing Service. This document establishes operating policy and procedures for the Computer Systems Laboratory's (CSL) testing program for Federal Information Processing Standards (FIPS) 152, Standard Generalized Markup Language (SGML), parsers. The testing methodology is based on ANSI X3.190-1992, Text and Office Systems - Conformance Testing for Standard Generalized Markup Language Systems. This document contains operating policy for an SGML Conformance Testing Service and is not intended to explain the detailed procedures that can be found in the documentation associated with the SGML parser validation system, commonly called the SGML Test Suite. 



\section{Table of Contents}

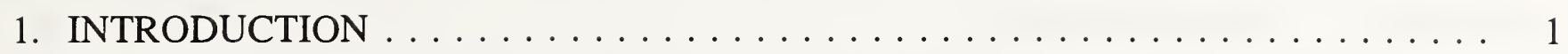

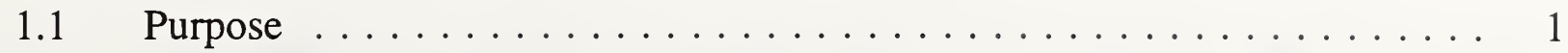

1.2 Background ......................... 1

1.3 Validated Products List (VPL) . . . . . . . . . . . . . 1

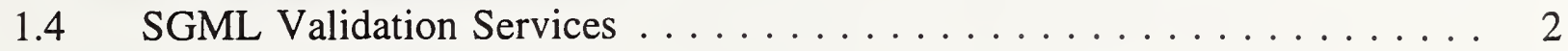

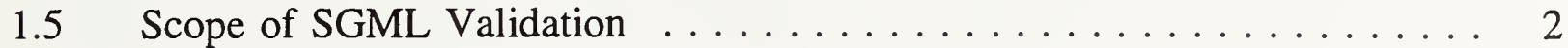

1.5.1 Identification of Environment Tested . . . . . . . . . . 3

1.5.2 Caveats ........................ 3

$1.6 \quad$ Role of Procuring Agency $\ldots \ldots \ldots \ldots \ldots \ldots \ldots \ldots \ldots \ldots \ldots \ldots$

2. REGISTRATION OF VALIDATION RESULTS $\ldots \ldots \ldots \ldots \ldots \ldots \ldots \ldots$

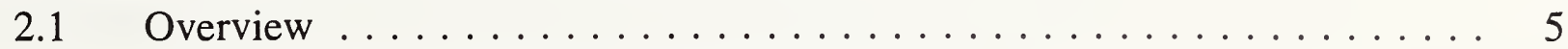

2.2 Specification of Validation Platform $\ldots \ldots \ldots \ldots \ldots \ldots$

2.3 Validation Summary Report $\ldots \ldots \ldots \ldots \ldots \ldots \ldots \ldots$

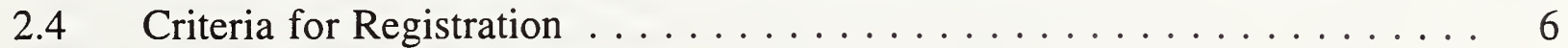

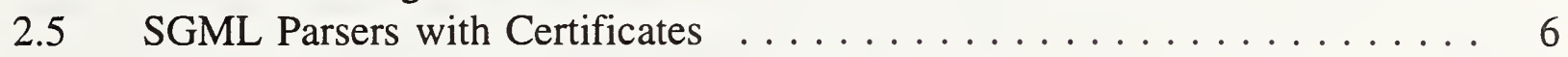

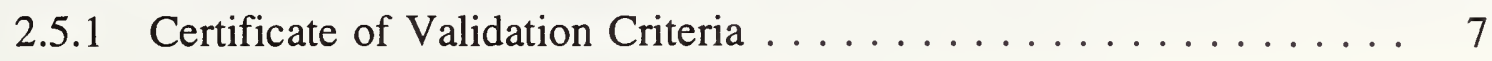

3. SGML VALIDATION PROCEDURES $\ldots \ldots \ldots \ldots \ldots \ldots \ldots \ldots \ldots \ldots$

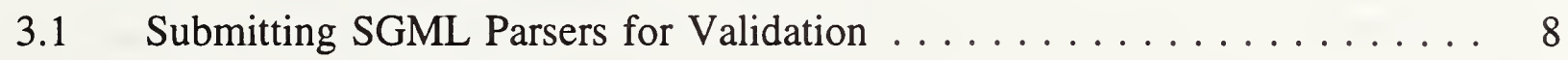

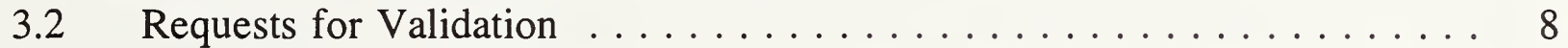

3.2.1 Typical Validation Schedule . . . . . . . . . . . . . . 9

3.3 Disputed and Withdrawn Tests $\ldots \ldots \ldots \ldots \ldots \ldots \ldots \ldots \ldots \ldots$

3.4 Funding (Reimbursement) $\ldots \ldots \ldots \ldots \ldots \ldots \ldots \ldots \ldots \ldots \ldots \ldots$

3.5 Release of Validation Information . . . . . . . . . . . . . 11

3.5.1 Publication and Proprietary Data $\ldots \ldots \ldots \ldots \ldots \ldots \ldots \ldots$

3.5.2 Proprietary Data . . . . . . . . . . . . . . . . . 11

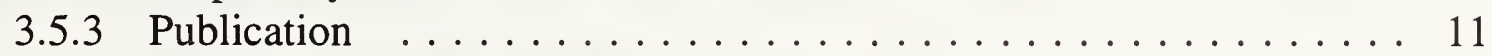

4. SGML PARSER VALIDATION SYSTEM . . . . . . . . . . . . . . . . 13

$4.1 \quad$ SGML Test Suite and Release Schedule . . . . . . . . . . . . . . . 13

APPENDIX A - NOTICE OF REGISTERED VSR . . . . . . . . . . . . . . . . . 14

APPENDIX B - SGML VALIDATION REQUEST FORM . . . . . . . . . . . 15

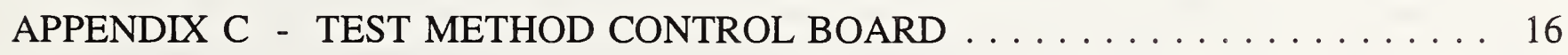




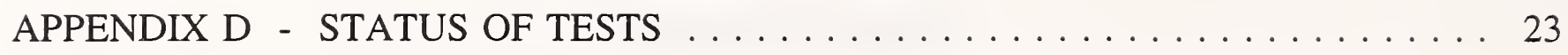

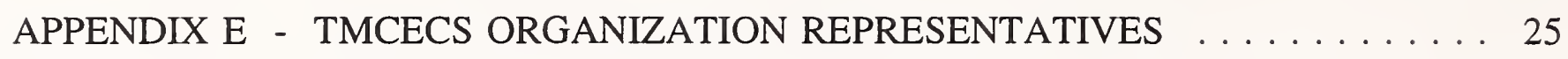

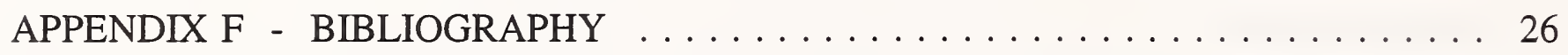




\section{INTRODUCTION}

\subsection{Purpose}

This document establishes operating policy and procedures for the Computer Systems Laboratory's (CSL) validation program for Federal Information Processing Standards (FIPS) 152, Standard Generalized Markup Language (SGML) [1], parsers. The testing methodology is based on ANSI X3.190-1992, Text and Office Systems - Conformance Testing for Standard Generalized Markup Language Systems [2]. This document contains operating policy for an Standard Generalized Markup Language [3] Conformance Testing Service and is not intended to explain the detailed procedures that can be found in the documentation associated with the SGML parser validation system, commonly called the SGML Test Suite.

\subsection{Background}

The Computer Systems Laboratory of the National Institute of Standards and Technology (NIST) is responsible for establishing conformance testing programs for Federal Information Processing Standards (FIPS). In carrying out this responsibility, CSL specifies the necessary conformance test specifications, test methods (i.e., test suites, test tools, and technical procedures), validation procedures, and testing laboratories for testing product compliance to FIPS.

The CSL conformance testing program for SGML provides for the testing of SGML parsers for conformance to the FIPS. Testing of SGML parsers to determine the degree to which they conform to the Federal Standards may be required by Government departments and agencies in accordance with the FIPS, the Federal Information Resources Management Regulation (FIRMR) 201-20.303, 201-20.304, and 201-39.1002, and the associated Federal ADP and Telecommunications Standards Index (hereafter referred to as the Index). The testing information available from CSL aids Federal procurement authorities, as well as industry, in determining if the SGML parsers offered to the government comply with the Government's requirements for FIPS.

The testing information provided by the SGML Conformance Testing Service is published in Certificates of Validation, Validation Summary Reports (VSR), Registered Validation Summary Reports, The Validated Products List (VPL), and validation procedures.

\subsection{Validated Products List (VPL)}

CSL publishes, on a quarterly basis, a Validated Products List that is a collection of registers describing implementations of Federal Information Processing Standards that have been validated for conformance to FIPS. The VPL contains information about the organizations, test methods, and procedures that support the validation programs for the FIPS identified in this document. For example, the VPL contains information about computer security, programming languages, SQL, Portable Operating System Interface for Computer Environments (POSIX), Government Open Systems Interconnection Profile (GOSIP), etc. When appropriate, SGML will be added to the VPL. 
For SGML, the VPL will identify the SGML parsers and the computer system environment on which the validation testing took place.

The VPL is intended to serve as an index to more detailed information. Each SGML entry in the VPL will be a very limited extract from a VSR available from CSL. The VSR will also be available from the testing laboratories.

\subsection{SGML Validation Services}

The SGML Validation Service will use testing laboratories to provide a testing service to evaluate conformance of SGML parsers to requirements of FIPS 152 (FIPS SGML).

Prior to validation, the SGML Test Suite is purchased by the SGML supplier (vendor) from CSL. The SGML supplier performs self-testing to identify and correct nonconformities. A formal validation, at a latter date, consists of an audited execution of the same tests cases. The format of the validation is a live test demonstration by the supplier's staff at the supplier's site or at the testing laboratory. The testing laboratory staff must review and approve the environment and proposed method of execution for the live test demonstration. The testing laboratory staff then audits the live test demonstration and documents the results in a VSR. The timing of the validation is controlled by a series of rigorous procedures detailed within this manual. The SGML supplier must provide technical support personnel for the validation service.

The SGML Test Suite is a package containing over one thousand test scripts. In order for an SGML parser to conform to FIPS 152, the SGML parser must be validated by passing all of these test scripts.

\subsection{Scope of SGML Validation}

SGML validation has a very narrow scope. The results of validation apply only to the SGML parser tested, its operating environment, and the SGML Test Suite version used. The Certificate of Validation verifies that the product has been tested using the Official National Institute of Standards and Technology SGML Conformance Test Suite for the Federal Information Processing Standards Publication 152 and that the test results obtained have been validated by NIST. Additional features of the SGML product are not being tested. Therefore, a successfully validated product that receives a certificate only implies that the SGML parser within the product passed all the tests thereby demonstrating its conformance with FIPS 152.

The output from running the test scripts must be compliant with ANSI X3.190-1992, Text and Office Systems - Conformance Testing for Standard Generalized Markup Language (SGML) Systems. The SGML product must output the parser results from running the test scripts in a Reference Application for SGML Testing (RAST) format. RAST interprets all markup to allow for machine comparison of test results for documents conforming to ISO 8879. RAST indicates in a standard way when a tag, processing instruction, or data is recognized by the parser, replacing references and processing markup declarations and marked sections appropriately. 
The results should be reproducible for the environment tested, but not necessarily for other environments. Therefore, it is important to document accurately the significant software and hardware components involved.

\subsubsection{Identification of Environment Tested}

The supplier of an SGML parser will identify the SGML parser (and components), the operating system, and the hardware systems to be used in a given validation. Version and release numbers, as well as model numbers, will be identified as appropriate. All of this information is provided by the supplier on the validation form "SGML Validation Request Form" (See Appendix B). This information is confirmed, to the extent possible, at the time of the validation. The VSR written by the testing service will additionally identify the SGML Test Suite version number and the interfaces, features, or profiles tested as well as the test results. The VSR will document configuration options, parameters, and any special procedures used in validation testing.

\subsubsection{Caveats}

Validation testing does not warrant that the product tested will perform according to all the vendor's assertions. Validation only specifies that the parser within the vendor's product was tested and that the test results passed the requirements of FIPS 152.

Validation testing does not warrant that the product tested is free of nonconformities, even if all tests are passed. The practical goal of SGML validation is to identify SGML parsers which may be procured (by federal agencies) and used to develop application programs which meet the FIPS goals of portability and interoperability.

The SGML Test Suite is not designed to replace the vendor's quality assurance testing or systematically to detect inconsistencies or "bugs." The primary technical goal of SGML validation testing is to verify that the SGML product correctly supports all required features of FIPS 152. Rather than exhaustive testing of permutations of features, the SGML Test Suite contains a carefully-chosen set of test cases which cover the required syntax and demonstrate the correct implementation of each of the applicable general rules of the standard.

Validation is not intended as a means of performance benchmarking. The VSR does not contain information about the speed, cost, or efficiency of executing the validation programs.

Validation is not intended to evaluate all optional features of the standard. For the SGML standard, optional features are allowed but will not be tested except for the two required by FIPS 152: OMITTAG and FORMAL.

\subsection{Role of Procuring Agency}

The procuring agency has the authority to extrapolate from the test results published in the VPL to other hardware/software environments, based on additional research by the procuring agency, 
demonstrations or warranties by the SGML supplier, etc. The procuring agency, not CSL, has the responsibility of reviewing the SGML validations listed in the VPL and determining the applicability of these validations to the hardware/software environment involved in a specific procurement. The criteria for applicability of a VSR or Certificate should be appropriate to the size and timing of the procurement. 


\section{REGISTRATION OF VALIDATION RESULTS}

\subsection{Overview}

Validation testing consists of a third party reviewing the supplier's testing procedures, witnessing the running of the conformance tests, evaluating the test results, and reporting the results of that testing in a VSR. If the validation procedures are followed and the VSR shows that the tested product demonstrated conformance to the standard (as defined by the test method and these validation procedures), a Certificate of Validation and Registered VSR is issued for the SGML parser on the computer and operating system tested.

A Certificate of Validation is issued if all required tests are passed and if all the criteria in Section 2.5.1 are met. SGML parsers with a Certificate of Validation are viewed as being "validated" at the FIPS level/option/profile that testing was performed.

\subsection{Specification of Validation Platform}

For validation testing, the SGML parser and its environment are specified as follows:

a. The name of the SGML parser (product) and its version or release number. [The version or release number specifies, at a minimum, the number under which the product is identified for marketing purposes. The number need not specify digits at the bug-fix level, unless they are relevant to SGML conformance.]

b. The name of the individual SGML components (and version or release numbers) used in the validation.

c. The name of the operating system and its version or release number.

d. The name of the computer hardware, including series and model numbers.

All of this information is provided by the supplier on the validation form "SGML Validation Request Form." This information is confirmed, to the extent possible, at the time of the validation.

The version of the SGML Test Suite and the date of the most recent maintenance change to the test suite are documented.

\subsection{Validation Summary Report (VSR)}

The results of validation testing are documented in a VSR. In addition to a specification of the validation platform, the VSR contains:

a. a table showing the pass/fail results in various categories for each applicable test suite type, b. documentation of changes made to the SGML test suite in order to install implementation- 

defined SGML language,
c. a description of test results for informational tests.

The information for the VSR is based primarily on test results obtained by a CSL recognized testing laboratory, witnessing the execution of the SGML Test Suite on the supplier's SGML parser. The VSR also contains information from statements made by the supplier concerning identification of the product, SGML parser options, and the operating environment in which validation testing was conducted.

Each VSR documents the execution of the entire test suite on at least one validation platform. If appropriate, validation results for multiple platforms with identical results (with respect to conformance testing) will be packaged into one document.

\subsection{Criteria for Registration}

The VSR may be registered with CSL if the following criteria are met:

a. The SGML parser has been tested with the latest official release of the SGML Test Suite.

b. The validation was performed by a CSL recognized testing laboratory.

c. All validation procedures and processing steps have been completed, including review and release of the VSR by the testing laboratory.

d. A final VSR has been prepared reporting the results of validation testing.

e. The SGML parser is a released (commercially available) product or this is the first time the pre-release product has been tested. Pre-release products may be validated only once, and revalidation is required to remove the "Pre-release" designation.

There is only one category under which SGML parsers will be listed in the VPL: SGML Parsers with Certificates.

\subsection{SGML Parsers with Certificates}

A CSL Certificate of Validation is issued if the SGML parser tested demonstrates conformance to the respective FIPS based on the SGML Test Suite. The Certificate of Validation identifies the SGML parser tested, the version of the applicable SGML Test Suite, the FIPS (including applicable level) tested, the test suite types executed, and the hardware/software environment on which the SGML parser was tested.

The Certificate of Validation is valid regardless of subsequent SGML Test Suite releases. 


\subsubsection{Certificate of Validation Criteria}

A CSL Certificate of Validation is issued for an SGML parser if the SGML parser passed all required SGML Test Suite tests for the applicable test suite type(s). The SGML parser must also meet the criteria for registration in Section 2.4. 


\section{SGML VALIDATION PROCEDURES}

\subsection{Submitting SGML Parsers for Validation}

The FIRMR refers to the Index that provides terminology for agencies to use when incorporating FIPS in Government procurements. This terminology requires that SGML parsers entering the Federal Government inventory be validated. This requirement may be satisfied by supplying a current Certificate of Validation for the SGML parser, or, at the option of the procuring agency, temporarily satisfy this requirement by submitting the SGML parser for validation.

The phrase "submission for validation" as used in the Index means that a letter has been received by the test laboratory requesting that the SGML parser be validated for the purposes of offering the SGML parser to the Government. When such a request is received, the test laboratory will send the requestor a letter acknowledging receipt of the request and indicate the month in which validation testing is scheduled to take place. This letter may be offered to Government departments and agencies as proof of submission for validation.

The SGML parser remains submitted for validation so long as all information necessary to complete the validation process is provided before the end of the month previous to the scheduled validation.

If the information is not provided by the end of the month previous to the scheduled validation, and if an alternate schedule has not been specified in writing by the test laboratory, then the requestor will no longer be considered scheduled. To be rescheduled the requestor must submit a letter requesting a new validation date.

\subsection{Requests for Validation}

A request for validation services shall be in the form of a letter to any of the approved test laboratories. NIST may be contacted at the following address to receive a list of the approved test laboratories:

National Institute of Standards and Technology (NIST)

Computer Systems Laboratory

Systems and Software Technology Division

Office Systems Engineering Group

Building 225, Room B266

Gaithersburg, Maryland 20899 (U.S.A.)

Ronald Wilson

Telephone (301) 975-3352 (Direct line)

(301) 975-3345 (Secretary)

(301) 926-3696 (FAX)

e-mail: wilson@sst.ncsl.nist.gov (INTERNET)

The following information should be provided:

a. Desired and alternate month of testing,

b. SGML parser identification,

c. Point of contact for validation, and 
d. Statement specifying whether or not the request is submitted for validation for the purpose of offering the SGML parser to the Government in response to a Request For Proposal (RFP).

The SGML Validation Request Form in Appendix B may be sent instead of the letter of request to provide the above information.

A request for validation of an SGML parser should be received by the test laboratory no later than 90 calendar days prior to the month desired for on-site testing. Requests for changes to an existing test date should be submitted no later than 30 days prior to the scheduled month of testing. Requestors may choose to have one or more SGML parsers validated at the same time, or may schedule them separately, according to their needs.

Upon receipt of a request for validation, the test laboratory will provide prevalidation materials (forms and lists) to the requestor. The test laboratory will also verify that the requestor has a current license to use the SGML Test Suite.

In order to guarantee timely validation, the required preliminary prevalidation material must be forwarded to the test laboratory 60 calendar days prior to the month of the scheduled on-site testing. Additional prevalidation material, as specified by the test laboratory, must be forwarded 30 calendar days prior to the month of the scheduled on-site testing. Upon receipt of the prevalidation material, an actual date within the scheduled month will be established for on-site testing. On-site testing dates will not be established for first time validations until after a successful review of prevalidation materials by the test laboratory.

All hardware, software (except for the SGML Test Suite), and technical support must be provided by the requestor. The test laboratory determines the location where the on-site testing will take place.

\subsubsection{Typical Validation Schedule}

The following is a typical schedule for a validation:

(Where "T" represents the day on-site testing begins and '-' or ' + ' integer represent the number of calendar days before or after the beginning on-site testing day.)

T - 90 Client purchases SGML Validation Test Suite

$\mathrm{T}-90 \quad$ Client contacts test laboratory to request validation services

$\mathrm{T}-60$ Client submits preliminary prevalidation materials and disputed tests, if any

$\mathrm{T}-30 \quad$ All prevalidation test materials received

$\mathrm{T}-30 \quad$ Resolve all outstanding issues and schedule on-site testing

$\mathrm{T} \quad$ On-site testing begins (scheduled month)

$\mathrm{T}+10 \quad$ Draft VSR available

$\mathrm{T}+30 \quad$ Final VSR available 


\subsection{Disputed and Withdrawn Tests}

If a supplier believes a test is in error, the supplier must send an error report to the test laboratory (who will forward it to CSL) with associated rationale and detailed documentation no later than 60 days before the on-site testing so that CSL can make a determination of the appropriate action. CSL will notify the supplier of the determination thirty days prior to the on-site visit. If the test is determined to be nonstandard, then it will be withdrawn from the SGML Test Suite, or it may be designated as an informational test for purposes of reporting in the VSR.

A Test Method Control Procedures Model will be employed in order to resolve disputes concerning the SGML Test Suite. The purpose of the Test Method Control Executive Committee (see Appendix D) is to resolve issues in a fair and speedy fashion. The committee is responsible for:

- Resolving challenges to the test method,

- Overseeing the maintenance of the test method,

- Coordinating issues of interpretations of the standard,

- $\quad$ Procedures and documentation associated with the test method,

- Advising on the proper use of the test method,

- Recommending changes to these procedures,

- $\quad$ Accepting/recognizing new test methods, and,

- $\quad$ Recommending improvements for the next revision of the test method.

Any technical issues that are not resolved between CSL and the supplier by the on-site date may be submitted by the supplier for formal interpretation to the appropriate Standards Committee (e.g., SC18, Working Group 8) or to CSL in accordance with the latest version of FIPS PUB 29, "Interpretation Procedures for Federal Information Processing Standards for Software." FIPS PUB 29 is available from the National Technical Information Service (NTIS), Springfield, VA 22161, telephone number (703) 487-4750. If formal interpretation is requested from a Standards Committee, CSL must receive a copy of the request for interpretation and all other relevant correspondence.

If a dispute is not resolved at the time of on-site testing, and if CSL judges the dispute to have merit, the test will be listed in the VSR under the category "Tests Under Review." The test result (pass or fail) will be included in the VSR as an informational test. If the interpretation finds that the test is valid, the test will be reinstated to the SGML Test Suite, and the supplier will be informed of the interpretation results. CSL will also assign a date for the correction of the nonconformity, typically six months later (to facilitate auditing by a subsequent validation).

Other unresolved disputes will be listed as nonconformities in the VSR. If a determination is later made (in favor of the SGML parser) that the disputed test is in error, the VSR will be corrected and reissued and, if necessary, a Certificate of Validation will be issued. Any appropriate changes will be made in the next issue of the VPL. 


\subsection{Funding (Reimbursement)}

The testing laboratory will determine the fee for the validation service. An SGML Validation Request Form will be submitted to the requestor approximately 90 calendar days prior to the month of the scheduled on-site testing. A signed copy of the SGML Validation Request Form together with payment for the total cost of the validation must be returned to the testing laboratory no later than 60 calendar days prior to the month of the scheduled on-site testing. No work will be performed until there is assurance that funding is available for the validation. The CSL administrative fee of $\$ 1000.00$ per test will be submitted by the testing laboratory to CSL for the evaluation of the test results. This fee will be included within the fee the testing laboratory charges for the validation service.

\subsection{Release of Validation Information}

Until a VSR is registered, requests for information concerning validation of an SGML parser will be directed to the supplier.

\subsubsection{Publication and Proprietary Data}

In general CSL shall have the right to use all information gathered in the course of developing and administering a conformance testing program for any governmental purpose, but shall not release such information publicly except: (1) When reporting on the results of testing, CSL may provide information, subject to the provisions of the subparagraphs below; and (2) as required pursuant to a request under the Freedom of Information Act (5 U.S.C. Section 552).

\subsubsection{Proprietary Data}

The supplier of information shall place a Proprietary notice on all information delivered to CSL that the supplier asserts is proprietary. Any information designated as proprietary that is furnished to CSL, shall be used by CSL only for the purpose of carrying out validations. Information designated as proprietary shall not be disclosed, copied, reproduced or otherwise made available in any form to any other person, firm, corporation, partnership, association, or other entity without the consent of the supplier except as such information may be subject to disclosure under the Freedom of Information Act (5 U.S.C. 552). CSL will use its best efforts to protect information designated as proprietary from unauthorized disclosure. CSL will not be liable for the disclosure of information designated as proprietary that, after notice to and consultation with the supplier, CSL determines may not lawfully be withheld or that a court of competent jurisdiction requires disclosed.

\subsubsection{Publication}

VSRs registered with CSL shall be made available to the public. In no event, however, shall the name of the supplier or any of its trademarks and trade names be used in CSL publications without the supplier's prior written consent. With respect to publication in the VPL, the standard Validation Customer Agreement shall contain the supplier's written consent. 
CSL and the supplier shall agree to confer and consult prior to the publication of data to assure that no Proprietary Data is released and that patent rights are not jeopardized. Prior to publishing a VSR, the supplier shall be offered an opportunity to review such proposed publication. 


\section{SGML PARSER VALIDATION SYSTEM}

The Director of CSL, or designated representative, determines the SGML Parser Validation System (SGML Test Suite) version or release to be used for validation.

The NIST SGML Test Suite, Version 1.0, becomes the official version of the SGML Test Suite on January 1, 1995. To the best of our knowledge, this SGML Test Suite evaluates conformance to the minimum requirements of FIPS 152.

The NIST SGML Test Suite may be licensed from CSL. To request a copy of the SGML Test Suite and/or to submit questions regarding the SGML Test Suite, contact:

National Institute of Standards and Technology (NIST)

Computer Systems Laboratory

Systems and Software Technology Division

Office Systems Engineering Group

Building 225, Room B266

Gaithersburg, Maryland 20899 (U.S.A.)

Ron Wilson

Telephone (301) 975-3352 (Direct line)

(301) $975-3345$ (Secretary)

(301) 926-3696 (FAX)

e-mail: $\quad$ wilson@sst.ncsl.nist.gov (INTERNET)

\subsection{SGML Test Suite and Release Schedule}

A new version of the SGML Test Suite will be issued when warranted by significant changes. Changes may be required to the SGML Test Suite to correct existing errors, enhance test routines, and reflect SGML language interpretations. As per procedures defined within Appendix D, additional tests for features of SGML will be developed and added to the SGML Test Suite. Also, when a new version of the SGML standard is issued, and the FIPS is revised, the SGML Test Suite will normally be enhanced to test for the new features.

When a licensee requests SGML parser validation on either a new product or a different platform, CSL will distribute the latest version of the validation test suite for an additional charge equal to twenty percent of the original fee. 


\section{APPENDIX A - NOTICE OF REGISTERED VSR}

\section{Notice of Registered Validation Summary Report (VSR)}

$$
\text { January 10, } 1994
$$

The information below will be listed on the Computer Systems Laboratory (CSL) register of tested SGML Parsers.

The SGML parser identified in this Validation Summary Report (VSR) is considered to be "validated" because it did pass all required tests for a certificate and met the criteria for VSR registration as specified in the SGML Parser Validation Procedures. The VSR should be reviewed for identification of test environment parameters, implementation-defined specifications, withdrawn tests, and tests under review.

Report Issued to: SGML-Vendor, Inc.

VSR Number: NIST-94/7011

Vendor Representative: Ms. Beatrice Kim

SGML Parser Identification: SGML-202, Version 3.0 Pre-release

Validation Environment:

SGML Components: SGML-202 Engine, Version 3.0 Pre-release

Hardware: CHIP486, Model 1124

Operating System: CHIP-Ux, Version 5.0.3

Test Date: January 3, 1994

FIPS PUB: 152 SGML, September 26, 1988

Validation System: NIST SGML Test suite, Version 1.0

Approved Changes Dated: December 2, 1993

Tests Under Review: None

Features and Interfaces Tested:

Conformance to SGML ISO 8879-1986

Support for FIPS 152

Signed:

Roger Martin

Chief, Division 872 


\section{APPENDIX B - SGML VALIDATION REQUEST FORM}

\section{SGML VALIDATION REQUEST FORM}

SGML ( FIPS 152)

Company Name

Address

1. SGML Software Product to be validated: Name/Version \#

2. Test Environment: Hardware ID Model \# Operating System ID/Version \#

3. Validation Location:

(if different from above)

4. Validation month: Desired month Alternate Month

Deadline (if any) for completion of validation

Validation is requested for a specific RFP (yes/no)?

5. Points of Contact:

Contractual

Technical

Telephone

Telephone

This is not a commitment for a NIST validation. The undersigned will be contacted by NIST to discuss procedures, scheduling, and cost.

Signature

Date

(printed name) Phone 


\section{APPENDIX C - TEST METHOD CONTROL BOARD}

\section{TEST METHOD CONTROL EXECUTIVE COMMITTEE (TMCEC)}

Based on the document 'Test Method Control Procedures Model' [4], the following is an analysis of how such a model should be set up and implemented with regard to the day to day running and maintenance of the Standard Generalized Markup Language (SGML) validation service and its software.

Within the following document the words 'Test Method' refer to the SGML Test System and it's associated documentation.

\subsection{Organization}

The purpose of the TMCEC for SGML (hereinafter referred to as TMCECS) is to resolve issues in a fair and speedy fashion. Communications between members will be by electronic mail, and in cases where members have no access to this media, facsimile copy.

\subsubsection{Confirmation of Chairperson}

The chairperson shall oversee the running of the TMCECS and shall be the designated representative of NIST. The chairperson shall be the designated technical authority and shall have up to date knowledge of the status of the service and suites used within it.

\subsubsection{Designation of a Secretary}

The role of secretary in the TMCECS shall be held by, or appointed by, the chairperson.

\subsubsection{Establishment of committee voting procedures}

The voting procedure for all issues brought before the TMCECS is as follows:

- $\quad$ An issue is raised with any member of the TMCECS.

- The issue is brought to the attention of the chairperson.

- The chairperson forwards the issue to the members of the TMCECS (by fax or e-mail) for voting.

- The members of the TMCECS shall have 10 working days to return their vote on the issue in question. Votes can only be YES or NO. A non-returned vote is counted as a YES.

- $\quad$ No votes will be reviewed and the rationale sent to the TMCECS members. Members will have 5 working days to change their votes.

- A resolution to an issue in question is accepted after $75 \%$ of the members have voted in it's favor. 
- The result of the vote is made known.

\subsubsection{Establishment of Membership}

The membership of the TMCECS will be open to all test laboratories offering a SGML test service. The offers will be given to the following organizations (see Appendix E).

- NIST

- $\quad$ EXOTERICA CORPORATION

It may be decided that two vendor representatives be allowed to participate, to represent the client base in decisions with the proviso that commercially sensitive material will be sanitized before being discussed.

1.1.5 Establishment of an appropriate Interface Mechanism with the Standards Authority.

The interface mechanism with the standards authority may be the designated member of the TMCECS who also is a member of the ISO standards committee.

\subsection{Test Method Control Executive Committee}

The committee is responsible for:

- $\quad$ Resolving challenges to the test method.

- $\quad$ Overseeing the maintenance of the test method.

- Coordinating issues of interpretations of the standard.

- $\quad$ Procedures and documentation associated with the test method.

- $\quad$ Advising on the proper use of the test method.

- $\quad$ Recommending changes to these procedures.

- Initially accepting/recognizing new test methods.

- $\quad$ Recommending improvements for the next revision of the test method.

1.2.1. Resolving challenges to the test method.

A situation arising in which a party disagrees with the way that the SGML validation system is maintained in either the actions of testing or in the test suite itself should be dealt with using the voting procedures described in Section 1.1.3 above.

As a result of submitting a challenge to the test method to the TMCECS, one of five resolutions may be made as indicated in Appendix D, 'Status of Tests.' 
1.2.2. Overseeing the maintenance of the test method.

NIST will oversee the maintenance of the test method. As changes occur to the ISO Standard and testing experience is gained, the test method and test suite may require updating. The TMCECS will vote on recommended changes as described in Section 1.1.3.

\subsubsection{The SGML Test Suite}

The SGML test suite is updated for two reasons:

- A major change is made to the existing suite through the addition of new tests against new functionality.

- The update of the suite is to incorporate code improvements by applying bug fixes to the existing code.

\subsubsection{SGML Test System User Guide}

At the time of every update to the SGML test software, the relevant User Guide is also updated to reflect any changes that have been made. During the course of the test software's lifetime, the User Guide may be found to contain typographical errors or inaccuracies. These are dealt with by issuing a page update. Each page of the User Guide carries a date, thus enabling the User to know that an update has been made to the page since the document was issued. Changes to this document should not need to go before the TMCECS unless deemed necessary by the chairperson.

\subsubsection{Test Reports}

NIST maintains a SGML Test Report Proforma. This report is supplied to all test labs. It is a requirement that to be a test laboratory, the laboratory will produce a test report that meets NIST specifications. All test reports shall contain the same information and be structured in the same way. In order to ensure that this is the case, all test labs should forward a sample copy of the final test report of a validation to the chairperson. Issues may be raised regarding the content of the test report.

\subsubsection{Certificates}

Only NIST may issue Certificates of Validation.

1.2.3. Coordinating issues of interpretations of the standard.

Disputes based on interpretations of the standard shall be dealt with in the method set down in Section 1.2.1.

If the TMCECS agree that an interpretation of the standard is required, the issue is sent to the appropriate Standards Committee for resolution through the appointed standards committee liaison organization.

1.2.4. Advising Testing Laboratories, clients and others on the proper use of the test method.

Each Testing Laboratory is responsible for advising their clients on usage of the test method. 
Advice to other test labs by NIST will be made on an informal basis, unless the advice may effect all labs in such a way that the procedures may be affected.

1.2.5. Recommending changes to these procedures.

The function of the TMCECS shall be reviewed, at least, once a year. The chairperson shall distribute a questionnaire in which each member will be asked if they feel any changes are needed to the existing practices.

1.2.6. Initially accepting/recognizing new test methods.

All new test methods shall be put before the board for voting. If accepted, the methods will be implemented at the first opportunity. A new method, if accepted, shall also be recognized by those members who voted against the issue.

1.2.7. Recommending improvements and enhancements for the next revision of the test method.

These should be submitted to the Secretary for review by the TMCECS.

\subsection{Parliamentary Authority}

Robert's Rules of Order or other established alternatives acceptable to the Committee shall govern all proceedings of meetings not addressed herein. 


\section{PROCEDURES}

Procedures are necessary to ensure that all users of the test method (Testing Laboratories, Clients, etc.) use the same test method at all times. The procedures are comprised of three primary areas:

- $\quad$ Test method maintenance;

- Disputes; and

- Technical assistance.

\subsection{Test Method Maintenance}

\subsubsection{Test Method Releases}

\subsubsection{Types of Releases}

There are two types of test method releases. They are the release of a new version of the test method or release of an update to an existing version of the test method through bug fixes. Updates to the existing version of the test method shall be for correcting errors in the test method.

\subsubsection{Frequency of Test Method Release}

New versions of the test method should not be released more frequently than once a year. New versions of the test method shall be approved by the Committee prior to release.

Updates to the existing version of the test method are issued as deemed necessary by the Committee.

\subsubsection{Testing of New Versions of the Test Method}

New versions of the test method are made available for field testing by designated beta test sites.

\subsubsection{Announcement of New Releases of the Test Method}

An announcement will be made when new releases of the test method are available. Ordering information and the effective date of use for official conformity testing will be included in this announcement.

\subsubsection{Use of New Releases}

At the time of release of a new version of the test method, an announcement is made indicating when it replaces the current version for formal validation. The pre-testing portion of the conformity testing process must be performed using the same release of the test method as that used during the on-site portion of the conformity testing process.

\subsubsection{Method for Changing the Test Method}

Users of the test method may request changes to any portion of the test method; e.g., test suite, testing support tools, or associated documentation. Requests for change shall be submitted in 
writing or electronic mail to the Secretary of the Committee or the testing laboratory conducting conformity testing. All requests received by a testing laboratory shall be forwarded to the Committee for resolution. Requests for test method changes should be submitted using the format of the Software Error Report provided in the SGML User Guide.

The Committee shall evaluate and resolve all requests for change of the test method. Changes to the test method shall be made in accordance with committee approval criteria (see Section 1.1.3). The changes shall be incorporated into the test method either in the bug fix or next release of the test method.

The Committee shall authorize the withdrawal of any test case which is found to be incorrect. In each case, the test case is withdrawn from the current version of the Test Method used for all testing performed subsequent to the date of withdrawal of the test case. A list of withdrawn test cases will be maintained by the Secretary. The list of withdrawn test cases shall be included in each test report. Withdrawn test cases do not count towards the results of testing.

\subsubsection{Impact of Interpretations on the Test Method}

The TMCECS shall be responsible for reviewing all interpretations published by the committee of the recognized standards body responsible for the development of the standard to determine any necessary changes to the test method resulting from the interpretations.

Any necessary changes to the test method resulting from interpretations of the Standard language or the Test Method will be processed in accordance with these procedures. (See Section 2.1.2, "Method for Changing the Test Method")

\subsubsection{Test Method Documentation}

Each release of the test method shall be accompanied by documentation for implementing and using the test method. This documentation includes User Guide, checksheets and instructions for the submission of Software Error Reports.

\subsubsection{Changes to the Test Method Control Procedures}

Changes to these procedures may be recommended and submitted to the Committee for consideration.

\subsection{Resolution of Requests for Interpretations}

Requests for interpretation should be submitted to the Secretary for distribution to the Committee membership for consideration and resolution. Requests for interpretation may be of two types:

- Interpretations of the Standard language.

- $\quad$ Interpretations of the test method.

Any test cases that may be affected by pending interpretations shall remain in the Test Method (as Tests Under Review) until action is completed on the interpretation. Such test cases may result in nonconformities being identified in the test report. 
The test report shall state that an interpretation is pending in these cases. Test cases subject to interpretation do not count toward the results of testing.

\subsubsection{Interpretations of Standard Languages}

Requests for interpretation concerning interpretation of the standard language specification will be submitted to the appropriate standards organization for resolution. Further processing of the resulting interpretation will be in accordance with these procedures. (See Section 2.1.3, "Impact of Interpretations on the Test Method")

\subsubsection{Challenges to the Test Method}

The Committee shall evaluate and resolve all challenges to the Test Method. These resolutions shall be approved in accordance with the committees' established rules for approval (See Section 1.1.3). Any changes to the Test Method resulting from the resolution of these challenges will be processed in accordance with these procedures. (See Section 2.1.2, "Method for Changing the Test Method")

\subsection{Technical Assistance}

The TMCECS for the test method shall provide technical assistance to the Testing Laboratories and other users in the implementation and use of the test method. The Testing Laboratories shall provide technical assistance to users of the test method. 


\section{APPENDIX D - STATUS OF TESTS}

\section{STATUS OF TESTS}

The following Appendix provides information of the status of programs and tests of the SGML test suite if they are submitted to the dispute process of the TMCECS.

Within this Appendix, the word 'program' or 'audit routine' are the same. A program consists of a number of 'tests'.

The TMCECS is in existence to solve disputes raised by parties regarding the SGML validation suite and the procedures that exist to support it. The major use of the TMCECS will be to solve disputes regarding the SGML suite itself. The method of doing this will always be the same - the client (or test laboratory) will submit a dispute to the TMCECS and a decision will be made that may affect the status of the test within the test suite. There are five possible results of doing this.

- $\quad$ No Change.

The TMCECS do not agree with the dispute raised and decide that no change is to be made.

- $\quad$ Bug Fix Issued.

The TMCECS agree with the dispute raised and issue a bug fix that will alter the audit routine in such a way that the number of tests expected to be attempted remains the same. Bug fixes falling under this category will generally involve the correcting of syntactic errors in the code.

Test Withdrawn.

The TMCECS agree with the dispute raised and withdraw a test from an audit routine. The test is withdrawn since the change required to be made to correct the audit routine cannot be addressed by a bug fix.

A test is withdrawn using a bug fix and will remain withdrawn until the next release of the test suite. This is so noted in the test report. A decision should be made before the next release of the SGML test system as to the final status of the test. Where possible, the problem surrounding the test should be dealt with and the test fixed before the release of the new suite. Where this is not possible the test should be given the final status of 'Test Deleted'.

Test Deleted.

If the TMCECS reach the conclusion that a test of the SGML test suite is no longer suitable for inclusion in the suite it must be deleted. During the life cycle of the suite this should be done by bug fix and at the time of the new release the code connected with the test in question should be deleted from the test suite. The expected number of tests to be attempted for that audit routine should be altered as appropriate. 
- $\quad$ Test Under Review.

The TMCECS classify a test as TUR if they decide to send it to the Standards Interpretation Committee. The test is counted as TUR until the response from the Committee is known.

For the purpose of validation, a TUR should be run and the result noted along with the information that the test is TUR. 
APPENDIX E - TMCECS ORGANIZATION REPRESENTATIVES

\section{TMCECS ORGANIZATION REPRESENTATIVE}

$\begin{array}{lll}\text { Representative } & \text { Mailing Address } & \text { email/Phone/FAX } \\ \text { Ron Wilson } & \text { NIST } & \\ & \text { Computer Systems } & \begin{array}{l}\text { rwilson@ nist.gov } \\ \text { (301)975-3352 }\end{array} \\ & \text { Laboratory } & \\ & \text { Guilding 225, Room B266 } & \\ & \text { USA } & \\ & & \\ \text { John McFadden } & \text { Exoterica Corporation } & \text { jrm@exoterica.com } \\ & \text { 1545 Carling Avenue } & \text { (613)722-1700 } \\ & \text { Suite 404 } & \\ & \text { Ottawa } & \\ & \text { Ontario, Canada } & \\ & \text { K1Z 8P9 } & \end{array}$




\section{APPENDIX F - BIBLIOGRAPHY}

[1] Standard Generalized Markup Language (SGML), Federal Information Processing Standards Publication, September 26, 1988.

[2] ANSI X3.190-1992: for Information Technology - Text and Office Systems - Conformance Testing for Standard Generalized Markup Language (SGML) Systems, American National Standard, 1992.

[3] ISO 8879: Information Processing - Text and Office Systems - Standardized Generalized Markup Language (SGML), International Organization for Standardization, 1986.

[4] Test Method Control Procedures Model, Document No. D109-07, Workshop on Harmonization of Programming Languages and Graphics Validations, March 17, 1994. 
